\title{
Interaction of Neurotransmitter Systems in the Hippocampus: A Study of Some Behavioral Effects of Hippocampal Sympathetic Ingrowth
}

\author{
Viswaprabhu Ayyagari, Lindy E. Harrell, and Dee S. Parsons \\ Departments of Neurology and Psychology, Veterans Administration Hospital and University of Alabama at Birmingham, \\ Birmingham, Alabama 35294
}

\begin{abstract}
Recent research has suggested that normal learning/memory may depend upon the balance between central noradrenergic and cholinergic systems. This hypothesis has particular relevance to the study of the neuronal rearrangement that follows cholinergic denervation of hippocampus. In this, peripheral noradrenergic fibers, originating from the superior cervical ganglion, grow into the hippocampus in response to lesions of the medial septal (MS) cholinergic cell bodies. To understand further the influence of hippocampal sympathetic ingrowth (HSI) on behavior, gustatory neophobia, passive avoidance (PA) learning, and open field activity were studied. Male Sprague-Dawley rats underwent one of four surgical procedures: MS lesions and sham ganglionectomy (ingrowth group; MS/HSI group), sham MS lesions and ganglionectomy (Gx group), MS lesions and ganglionectomy (noingrowth group; MS/Gx group), or sham MS lesions and sham ganglionectomy (CON group). Behavioral testing began 4 weeks following surgery. The time to acquire the PA task was similar among all groups; however, the initial latency to enter the dark chamber of the PA apparatus was longer, and the number of partial reentries greatest, for MS/HSI animals. Retention testing at $24 \mathrm{hr}$ revealed that MS/HSI animals were significantly impaired when compared to the CON and MS/ Gx groups. The MS/Gx and the CON groups demonstrated gustatory neophobia, preferring water to saccharine solution, while gustatory neophobia was absent in the MS/HSI and Gx groups. MS/HSI animals were found to be more active in the open field than the other groups. Biochemical studies revealed the expected loss of ChAT activity in the dorsal and ventral hippocampi of lesioned animals along with elevated levels of norepinephrine (NE) in the dorsal hippocampus of MS/HSI animals. It is proposed that the balance between NE and ACh may be extremely important for learning/memory.
\end{abstract}

Over the last three decades, a variety of neuronal reorganizations have been demonstrated to occur in response to CNS injury (Cotman, 1985). Efforts to study the effects of such rearrange-

\footnotetext{
Received Oct. 9, 1990; revised Apr. 22, 1991; accepted Apr. 25, 1991.

This work was supported by National Institute on Aging grant 2-PO1-AG0656904 and a Veterans Administration Merit Veterans Administration Review grant to L.E.H.

Correspondence should be addressed to Viswaprabhu Ayyagari, Department of Neurology, SC 810 , University of Alabama at Birmingham, Birmingham, AL 35294.

Copyright (C) 1991 Society for Neuroscience $0270-6474 / 91 / 112848-07 \$ 03.00 / 0$
}

ments on hehavior and/or CNS biochemistry have, however, been somewhat hampered because in many experimental situations the effects of tissue injury cannot be clearly delineated from the effects of the neuronal rearrangement. One model that overcomes this dilemma and clearly allows for control of the tissue injury is that of hippocampal sympathetic ingrowth (HSI). In this neuronal rearrangement, peripheral noradrenergic fibers, originating in the superior cervical ganglion (SCG) and usually confined to the cranial blood vessels, grow into the hippocampus in response to lesions of the cholinergic cell bodies within the medial septum (Crutcher, 1987). This model system has advantages for studying neuronal rearrangements since SCG can be removed at the time of medial septal (MS) lesions in some animals and not others, thereby allowing comparisons between animals with lesions and with or without sympathetic ingrowth. Thus, the effect of this neuronal rearrangement can clearly be differentiated from the effect of the CNS injury.

Madison and Davis (1983) have demonstrated that the noradrenergic fibers associated with HSI produce norepinephrine (NE), which continues to increase throughout the life of the animal. Furthermore, HSI has been found to alter both physiological (Barker et al., 1984) and metabolic (Harrell et al., 1986) activity within the hippocampus. The behavioral effects of HSI have also been studied. Our laboratory has previously demonstrated that HSI is detrimental to the relearning of a spatial memory task (Harrell et al., 1983, 1990a; Harrell and Parsons, 1988). Interestingly, however, once animals reacquire the task, removal of HSI has no further detrimental effects (Crutcher et al., 1983; Harrell et al., 1983). Moreover, ganglionectomy alone produces no alteration in learning behavior (Harrell and Barlow, 1986). These findings suggest that HSI, with its associated increased NE concentration, somehow modulates the systems that underlie recovery of learning.

The role of NE in learning and memory and other behaviors has been debated (Leslie et al., 1985). Depletion of forebrain NE is known to alter responsiveness to novel physical environments and novel objects (Mason et al., 1978) so that animals show less fear than would normally be expected. The literature has been discordant on how NE affects gustatory neophobia (Gallagher and Burwell, 1989), passive avoidance, and open field activity (Heybach et al., 1978; Kimble et al., 1979).

Since it is now established that HSI alters the recovery of a cholinergic-mediated spatial behavior, we examined how this neuronal reorganization affects behaviors thought to be related to central NE levels, such as gustatory neophobia, passive avoidance, and open field activity. 


\section{Materials and Methods}

\section{Subjects}

Fifty-four male Sprague-Dawley rats (initial body weight, 150-200 gm) were housed in a temperature- and light-controlled (12L:12D) colony. Animals were housed communally except when undergoing neophobia testing, during which time they were placed in individual hanging wire cages. One week after adaptation to the colony, animals underwent surgical procedures (see below). Behavioral testing (see below) was started 4 weeks after surgery. On completion of the behavioral testing, the animals were decapitated, and their brains were removed within $30 \mathrm{sec}$. The brains were dissected on ice to yield a septal block for histology (see below), two hippocampi (which were further dissected into dorsal and ventral regions), and two neocortical samples for biochemical analysis of norepinephrine (NE), protein, and choline acetyltransferase (ChAT) activity (see below). All tissue samples were stored at $-70^{\circ} \mathrm{C}$ until processed for biochemistry or histology.

\section{Surgical procedures}

$\Lambda$ nimals were randomly assigned to one of four surgical procedures: CON (sham MS lesion and sham ganglionectomy); MS/HSI (lesion of the medial septum and sham ganglionectomy: ingrowth group); Gx (sham MS lesion and ganglionectomy); MS/Gx (MS lesion and ganglionectomy: no-ingrowth group). All surgery was performed under ketamine (110 mg/kg, i.p.) and xylazene $(13 \mathrm{mg} / \mathrm{kg}$, i.p.) anesthesia, with neurosurgical and general surgical procedures performed during one anesthetic administration.

Medial septal lesions (nose bar, $-2.5 \mathrm{~mm}$; coordinates from bregma; AP $+0.8 \mathrm{~mm}$, ML $0 \mathrm{~mm}$, DV $-5.9 \mathrm{~mm}$ ) were produced by passing direct current $(3 \mathrm{~mA}$ for $15 \mathrm{sec})$ through a bipolar, Teflon-coated stainless-steel electrode (tip diameter, $1 \mathrm{~mm}$ ). Sham neurosurgical procedures were performed in the same manner except that no current was passed.

The superior cervical ganglion (SCG) was removed bilaterally via a ventral midline neck incision. Blunt dissection was performed to expose the bifurcation of the common carotid; the ganglia were then visualized and subsequently removed. Sham surgeries were performed in a similar manner except that the ganglia were left intact. Initial removal was assessed by examination for a Horner's syndrome (ptosis and myosis).

\section{Behavioral procedures}

Four weeks after surgery, the animals were tested on three behavioral paradigms, in the following sequence: passive avoidance (PA) memory task, open field activity, and gustatory neophobia.

Passive avoidance task. Inhibitory avoidance learning was assessed in two stages: a learning trial and a retention trial. Animals were tested on a Lafayette Instruments model 12970 passive avoidance device, which consisted of a runway $(25 \times 75 \mathrm{~cm})$ illuminated by a $60 \mathrm{~W}$ incandescent lamp, separated by a guillotine door from a black Plexiglas chamber $(46 \times 40 \times 17 \mathrm{~cm})$ with a grid floor through which a scrambled foot shock could be administered.

Learning trials were begun by placing each rat on the brightly lit runway facing away from the dark chamber with the guillotine door open. The animal was allowed to explore the runway freely and enter the dark chamber. Once the animal entered the dark chamber, a scrambled foot shock of $200 \mu \mathrm{A}$ was administered and remained on throughout the trial. To escape the foot shock, the animal was required to return to the runway. The trial was terminated when the animal remained continuously on the runway for $2 \mathrm{~min}$. Initial latency to enter (LTE) the dark chamber (in sec) and the total time to achieve learning criterion were measured. In addition, the number of partial reentries, defined as placing the front paws in the dark chamber, was recorded.

Retention trials were performed $24 \mathrm{hr}$ later. Essentially, they were performed in the same manner as the initial learning trials except that no foot shock was administered when the animal entered the chamber. The number of partial entries and the latency to enter were recorded up to a maximum of $300 \mathrm{sec}$.

Activity. General motor activity was measured in an open field apparatus consisting of a $36 \times 36 \mathrm{~cm}$ square divided into nine $12 \mathrm{~cm}^{2}$ blocks surrounded by a 19-cm-high wooden wall. Rats were individually introduced into the center of the open field, and activity was measured in $1 \mathrm{~min}$ intervals for a total of $5 \mathrm{~min}$. Both horizontal (defined as the number of hind limb crossings between blocks) and vertical (defined as the number of rearings) activity was assessed.

Neophobia testing. Following the avoidance and activity testing, the animals underwent $3 \mathrm{~d}$ of adaptation to individual hanging cages with free access to food. Water was provided in two graduated bottles attached to the left and right side of the cage. Three days later, the rats were introduced to a $23 \mathrm{hr}$ food and water deprivation regimen, in which the rats were allowed access to the graduated water bottles and food for $60 \mathrm{~min}$. Water consumption (in $\mathrm{ml}$ ) in the first $30 \mathrm{~min}$ period was recorded (Dunn and Everitt, 1987). Baseline measures of water intake were obtained for $4 \mathrm{~d}$ to ensure that rats were sampling from both bottles. On the fifth day, a $0.01 \%$ saccharine solution was substituted for water in one bottle. This was counterbalanced such that saccharine was presented on alternate sides to half the animals in each group.

\section{Biochemical procedures}

Tissue preparation. The hippocampal and neocortical sections were thawed and rapidly homogenized in $1 \mathrm{ml}$ of phosphate-buffered saline ( $50 \mathrm{~mm}$ PBS). A $100 \mu \mathrm{l}$ sample was homogenized in $900 \mu \mathrm{l}$ of $0.5 \%$ Triton-PBS for measurement of choline acetyltransferase (ChAT) activity, while another $100 \mu \mathrm{l}$ aliquot was homogenized in $900 \mu \mathrm{l}$ of $0.1 \mathrm{~N}$ $\mathrm{NaOH}$ for protein estimation. The remaining $800 \mu \mathrm{l}$ was diluted $(1: 4)$ with ice-cold $0.1 \mathrm{~N} \mathrm{HClO}_{4}$ containing an internal standard dihydrobenzylamine (DHBA) for HPLC determination of NE levels.

Choline acetyltransferase. ChAT activity was measured using the method of Fonnum (1969). Homogenates of brain regions were incubated in triplicate for $20 \mathrm{~min}$ at $37^{\circ} \mathrm{C}$ in a buffer containing ${ }^{14} \mathrm{C}$-acetyl$\mathrm{CoA}(0.2 \mathrm{~mm}$; Sigma), choline (10 mM), and eserine $(1 \mathrm{~mm})$. The reaction was terminated by extraction of the synthesized ${ }^{14} \mathrm{C}$-acetylcholine into an organic phase containing scintillation fluid.

Norepinephrine. Following homogenization, the sample was centrifuged at $7360 \times g$ for $20 \mathrm{~min}$, the supernatant was removed, and centrifuged through a $0.2 \mu \mathrm{m}$ filter. A $50 \mu \mathrm{l}$ aliquot was injected across a Dynamax analytical column $(4.6 \mathrm{~mm} \times 1 \mathrm{~mm} \times 10 \mathrm{~cm} ; 3 \mu \mathrm{m}$ RP18) with a standard catecholamine mobile phase $[0.02 \mathrm{M}$ citric acid, $0.02 \mathrm{M} \mathrm{NaI}_{2} \mathrm{PO}_{4}$ buffer containing $0.269 \mathrm{mM}$ EDTA combined in a ratio of $1.64: 1$ with $0.36 \mathrm{M}$ sodium octyl sulphate, $4 \%(\mathrm{v} / \mathrm{v})$ acetonitrile, and $1.5 \mathrm{ml}$ phosphoric acid, final $\mathrm{pH} 3.5$ ]. Separations of catecholamines were performed at a flow rate of $1.5 \mathrm{ml} / \mathrm{min}$ across a polyethylene/ carbon working electrode with an $\mathrm{Ag} / \mathrm{AgCl}$ reference electrode maintained at a potential of $+0.72 \mathrm{~V}$ (sensitivity, $2 \mathrm{nA} / \mathrm{V}$ ). Calculations were based on the ratio of the detector responses for the amines to that of the internal standard.

Protein estimation. Protein content was estimated by the method of Lowry et al. (1951).

\section{Histological procedures}

Coronal sections $(20 \mu \mathrm{m})$ were taken in duplicates at $100 \mu \mathrm{m}$ intervals throughout the septum on an $\mathrm{AO}$ cryostat and stained for cresyl violet and acetylcholinesterase (Naik, 1963). A third series of sections through the septum (every $300 \mu \mathrm{m}$ ) was processed for norepinephrine histofluorescence (De la Torre and Surgeon, 1976).

\section{Data analysis}

All data were analyzed using the appropriate model analysis of variance (unweighted-means ANOVA; SAS; Winer, 1962) followed by Duncan's multiple-range test for post hoc testing. When appropriate, Student's $t$ test was employed for comparisons between groups.

\section{Results}

\section{Histology}

Twenty-one rats were excluded from the study because of improperly placed lesions or death. Data from the remaining 33 animals (CON, $n=8$; MS/HSI: $n=8 ; \mathrm{Gx}, n=10$; MS/Gx: $n$ $=7$ ) were used for further analysis. Examination of the septal region in animals with lesions (i.c., MS/HSI and MS/Gx) revealed complete destruction of the cholinergic cell bodies within the MS area and in some cases, the vertical limb of the diagonal band of Broca (Fig. 1). For the most part, there was no injury to the lateral septum, fornix, or overlying cortical areas. In the CON and the Gx groups, there was limited gliosis along the electrode track, but no obvious injury to the medial septum. 


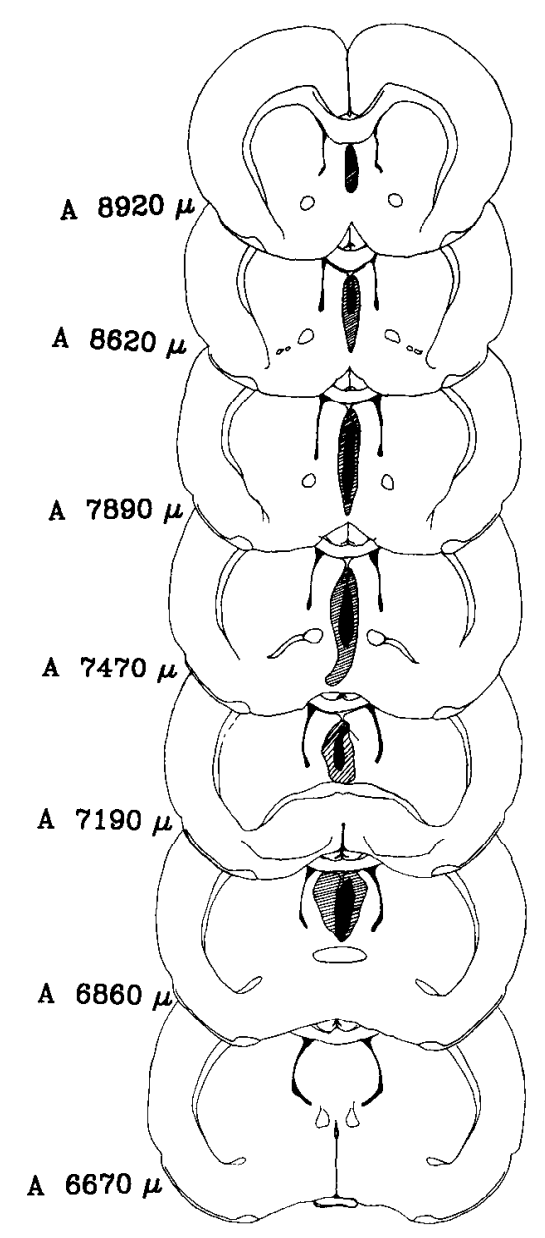

Figure 1. Schematic representation of lesion location and size from the MS/HSI and MS/Gx groups. Solid areas represent minimal lesion size, while hatched areas represent maximal lesion size.

As expected, peripheral NE fibers were observed on cerebral arteries and choroid plexus in both CON and MS/HSI groups, while these fibers were absent in the Gx and MS/Gx groups. These latter findings confirmed the completeness of SCG removal in both ganglionectomizcd groups.

\section{Behavioral tests}

\section{Passive avoidance experiments}

Learning trial. Table 1 presents the data for the initial learning phase of the PA experiment. Initial latency to enter the dark chamber was significantly affected by the various surgical procedures $\left(F_{(3,29)}=3.93 ; p<0.02\right)$. Thus, MS/HSI animals $(p<$ 0.05 , Duncan's) were found to enter the chamber later than
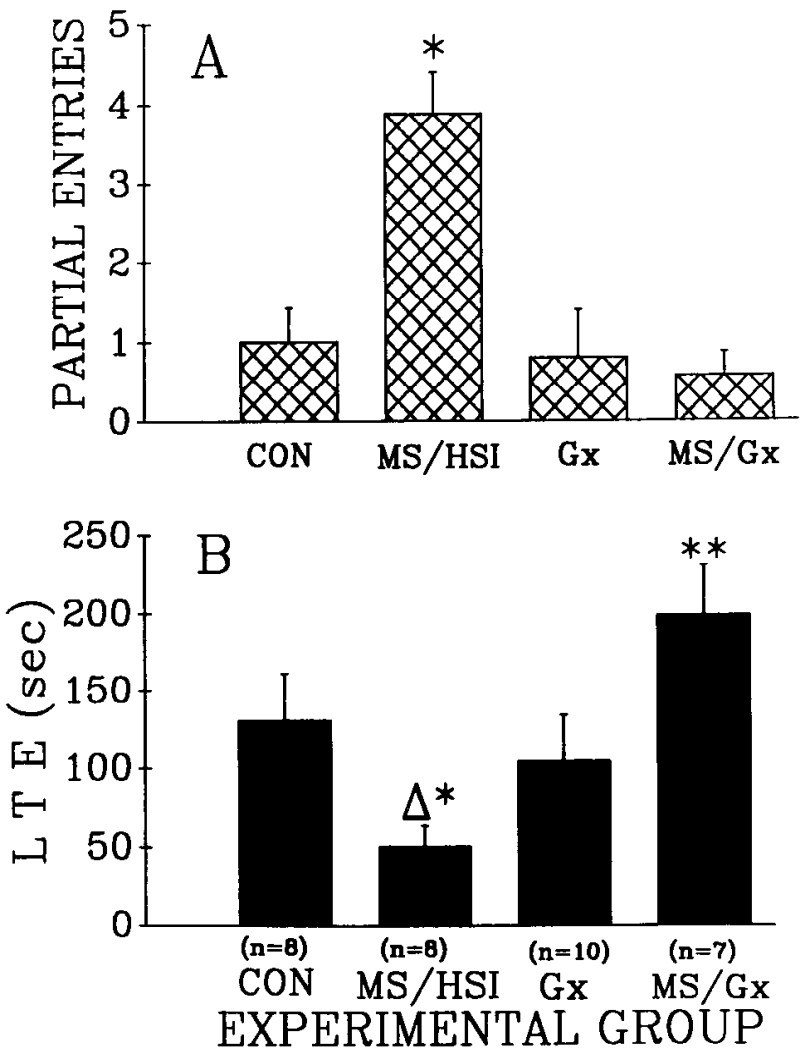

Figure 2. The effect of surgical procedures on performance of the PA task during the retention trial. The number of partial entries $(A)$ was significantly greater in MS/HSI group than other experimental groups $\left({ }^{*} p<0.05\right.$, Duncan's). Latency to enter $(B)$ was significantly shorter in the MS/HSI group when compared to CON $\left(\Delta^{*} p<0.05\right.$, Duncan's) and $\mathrm{MS} / \mathrm{Gx}(p<0.05$, Duncan's) groups. Gx animals wcre also found to differ from MS/Gx animals $\left({ }^{* *} p<0.05\right.$, Duncan's), but not from CON animals. Values are means \pm SEM. See Results for full statistical analysis.

$\mathrm{CON}, \mathrm{Gx}$, or MS/Gx animals, which did not differ from each other. In addition, MS/HSI animals also made the greatest number of reentries prior to achieving the learning criterion $\left(F_{(3,29)}\right.$ $=7.26 ; p<0.001)$. However, the total time to achieve learning criterion was not different among the groups.

Retention trial. In the retention phase, both the number of partial entries $\left(F_{(3.29)}=9.31 ; p<0.001\right)$ and $\operatorname{LTE}\left(F_{(3,29)}=4.57\right.$; $p<0.01$ ) were found to be dependent on the surgical procedures that the animals underwent (Fig. 2). Thus, the MS/HSI group, in comparison to the other three groups, was found to make the greatest number of partial entries ( $p<0.05$, Duncan's). Neither the CON, Gx, nor MS/Gx groups differed among themselves (Fig. 2A). The four experimental groups did differ significantly

Table 1. Effects of surgical procedures on initial learning of a PA task

\begin{tabular}{lccc} 
Group & LTE $(\mathrm{sec})$ & Reentries & Criterion time (sec) \\
\hline Con $(n=8)$ & $6.25 \pm 1.16$ & $5.13 \pm 1.16$ & $318.50 \pm 58.15$ \\
MS/HSI $(n=8)$ & $14.12 \pm 1.37^{*}$ & $12.87 \pm 2.21^{* *}$ & $472.80 \pm 86.06$ \\
Gx $(n=10)$ & $9.50 \pm 1.72$ & $3.80 \pm 1.16$ & $286.20 \pm 31.09$ \\
MS/Gx $(n=7)$ & $9.43 \pm 2.03$ & $4.00 \pm 1.27$ & $243.80 \pm 57.98$ \\
\hline
\end{tabular}

${ }^{* *} p<0.01,{ }^{*} p<0.05$, when compared with other groups. 


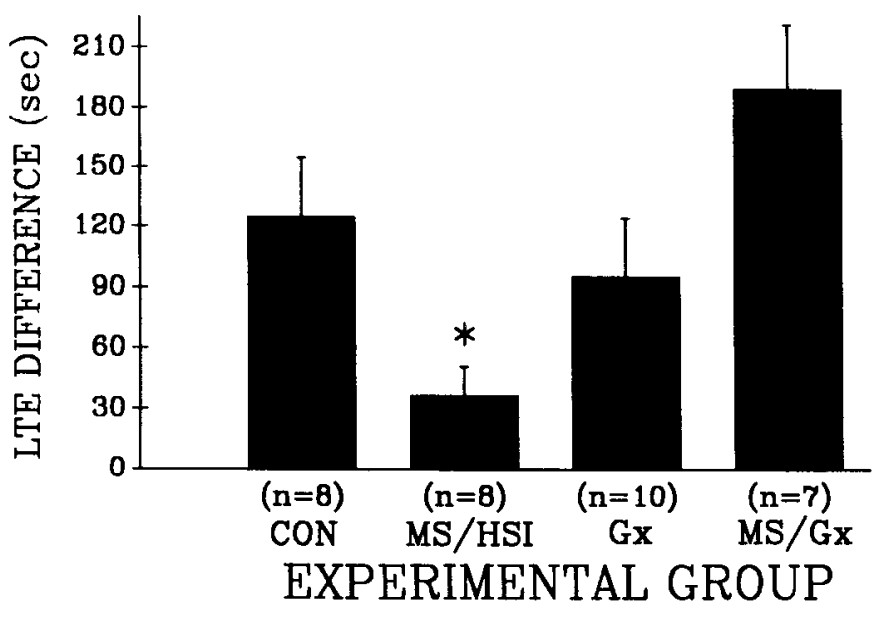

Figure 3. Latency to enter data expressed as the difference between the acquisition and retention trial. While there is a definite increase among all groups, the magnitude of change in the MS/HSI group is significantly less than in the other groups $\left({ }^{*} p<0.0001\right)$. Values are means \pm SEM. See Results for full statistical analysis.

on the length of time they withhcld cntry. Thus, LTE was found to be shortest for the MS/HSI group, followed by the Gx, CON, and MS/Gx groups (Fig. 2B). Statistical comparisons of LTE revealed that the MS/HSI group did not differ from the Gx group. However, LTE was significantly shorter in the MS/HSI group ( $p<0.05$, Duncan's) when compared to both CON and MS/Gx groups, which did not differ from each other. The LTE of $\mathrm{Gx}$ animals was not different from controls, but significantly longer ( $p<0.05$, Duncan's) than MS/Gx animals. To ascertain if MS/HSI animals retained information about the task, the difference between the initial and retention latencies to enter the dark chamber was determined. Figure 3 shows that MS/HSI animals did retain some information about the task, as there was a small difference between the two latencies. However, the magnitude of this change was significantly less than that seen in the other three groups $\left(F_{(3,29)}=6.69 ; p<0.01\right)$.

\section{Open field activity}

The groups differed significantly on the mean number of squares traversed in five $1 \mathrm{~min}$ intervals $\left(F_{(3,29)}=6.69 ; p<0.01\right)$, with the MS/HSI group ( $p<0.05$, Duncan's) traversing the greatest number of squares per minute (Fig. 4). The groups also differed significantly on number of rearings $\left(F_{(3,29)}=3.22, p<0.05\right.$; Fig. 4). While the groups showed a similar amount of rearing activity during the first $2 \mathrm{~min}$, it was observed during the 3-5 min period that the CON and $\mathrm{Gx}$ animals made less number of rearings than did the animals with the MS lesions. MS/HSI animals were consistently the most active ( $p<0.05$, Duncan's) in both horizontal and vertical movements.

\section{Gustatory neophobia}

During the baseline measurements, total water consumption was found to be similar among all groups of animals. Morcover, no specific side preferences were appreciated during this time period, as the animals sampled from both the graduated water bottles (data not shown).

The data on the neophobia testing are presented in Figure 5. While the groups did not differ in the total volume of fluid consumed in a $30 \mathrm{~min}$ period, there was a distinct pattern $\left(F_{(3,29)}\right.$

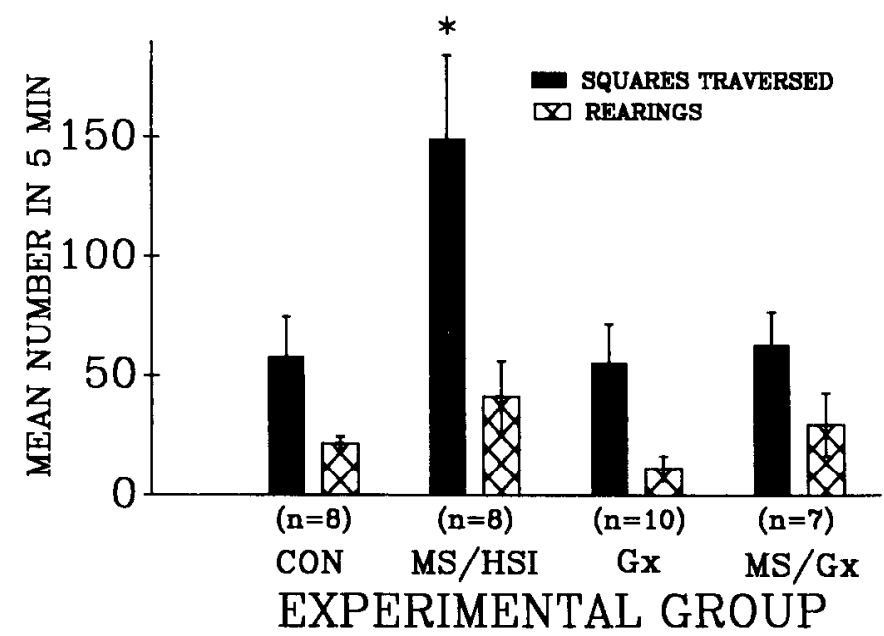

Figure 4. Horizontal and vertical activity on the open field task. Data are expressed as means \pm SEM during five $5 \mathrm{~min}$ trials. See Results for full statistical analysis.

$=15.23 ; p<0.0001)$ to the preference for the $0.01 \%$ saccharine solution, with the MS/HSI and the Gx animals preferring saccharine and the CON and MS/Gx animals preferring water. When the data on saccharine consumption were expressed as a percentage of the total fluid intake, the difference between the groups was even more striking $\left(F_{(3,29)}=31.95 ; p<0.0001\right)$. Thus, the MS/HSI and the Gx groups obtained approximately $75 \%$ of their fluid as saccharine, while the $\mathrm{CON}$ and $\mathrm{MS} / \mathrm{Gx}$ groups avoided this novel solution, consuming nearly $75 \%$ of their total fluid as water.

\section{Biochemical procedures}

Overall, the various surgical procedures were found to alter ChAT activity $\left(F_{(3,29)}=8.16 ; p<0.001\right)$, and NE levels $\left(F_{(3,29)}\right.$ $=3.08 ; p<0.05)$ in the dorsal hippocampus and ChAT activity $\left(F_{(3,29)}=5.46 ; p<0.005\right)$ in the ventral hippocampus. In the

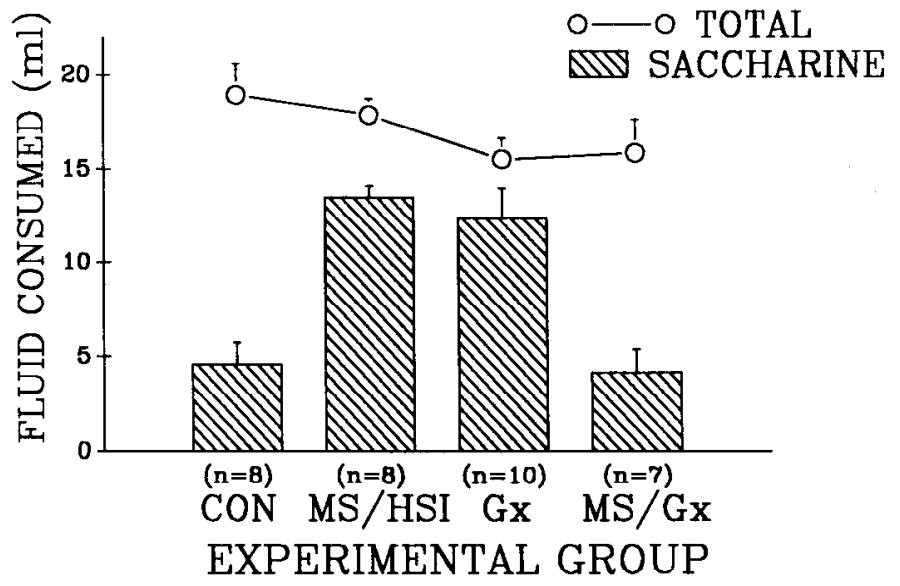

Figure 5. The effect of surgical procedures on gustatory neophobia. Absolute (mean \pm SEM) amounts of fluid (water and saccharine solution) and saccharine consumed during a $30 \mathrm{~min}$ period are represented. Note that the total fluid consumption was similar among groups; however, MS/HSI and $\mathrm{Gx}$ animals consumed significantly $\left({ }^{*} p<0.05\right)$ more saccharine than CON or MS/Gx animals. See Results for full statistical analysis. 
Table 2. Effects of surgical procedures on ChAT and NE in regions of the rat hippocampus

\begin{tabular}{|c|c|c|c|c|}
\hline \multirow[b]{2}{*}{ Group } & \multicolumn{2}{|c|}{$\begin{array}{l}\mathrm{ChAT} \\
\text { (nM/min/mg protein) }\end{array}$} & \multicolumn{2}{|c|}{$\begin{array}{l}\mathrm{NE} \\
\text { (ng/mg wet tissue) }\end{array}$} \\
\hline & Dorsal & Ventral & Dorsal & Ventral \\
\hline Con $(n=8)$ & $1.82 \pm 0.30$ & $1.96 \pm 0.39$ & $0.48 \pm 0.17$ & $0.91 \pm 0.39$ \\
\hline MS/HSI $(n=8)$ & $0.65 \pm 0.14^{* * *}$ & $0.74 \pm 0.23^{* *}$ & $4.31 \pm 1.77^{*}$ & $2.58 \pm 1.53$ \\
\hline $\mathrm{Gx}(n=10)$ & $1.55 \pm 0.23$ & $1.51 \pm 0.34$ & $1.82 \pm 0.99$ & $2.36 \pm 0.87$ \\
\hline $\mathrm{MS} / \mathrm{Gx}(n=7)$ & $0.71 \pm 0.16^{* * *}$ & $0.69 \pm 0.22^{* *}$ & $0.70 \pm 0.24$ & $2.91 \pm 1.15$ \\
\hline
\end{tabular}

neocortex, ChAT activity and NE levels were similar among the groups (data not shown).

Table 2 presents NE and ChAT data by group and anatomical region. Post hoc analysis revealed that animals with MS lesions, regardless of the presence or absence of HSI, had reduced ChAT activity in the dorsal and ventral hippocampus when compared to either CON or Gx animals ( $p<0.05$, Duncan's).

Not surprisingly, NE levels were found to be increased in the dorsal hippocampus of MS/HSI animals when compared to the other three groups ( $p<0.05$, Duncan's), which did not differ from each other.

\section{Discussion}

The behavioral paradigms employed in this study can be conveniently divided into tasks that require learning (passive avoidance) and those that are spontaneous (gustatory neophobia and activity on an open field). Our results suggest that HSI has a significant effect on both types of behavior. Thus, animals with HSI were found to be hyperactive, have an impairment in PA retention, and have an alteration in gustatory neophobia.

The PA task has been used as a behavioral task to model learning and memory in a variety of experimental paradigms, including those studying different forms of cognition (Slangen et al., 1990), cognition enhancers (Sanger and Joly, 1990), reinforcement and memory effects of neuropeptide substance $\mathrm{P}$ (Huston and Oitzl, 1989), changes in septohippocampal neurons in the aged rat (Lamour et al., 1989), neurobehavioral toxicity measurements (Bignami, 1987), animal models of Alzheimer's disease (Pepeu et al., 1986), stress-induced analgesia (Curzon et al., 1986), and the role of pituitary hormones in amnesia (Rigter and van-Riezen, 1979). Most PA studies have utilized a paradigm in which animals are allowed to make an initial association between environment and a particular event (e.g., foot shock) and are then retested $24 \mathrm{hr}$ later for retention of this initial association (Leslie et al., 1985). Deficits in retention of a PA task have been attributed to both failure of memory (Black et al., 1977) and failure of response inhibition (Douglas, 1967; Kimble, 1968; Livesey et al., 1984; Galey et al., 1985). When an animal demonstrates a performance deficit on the retention trial of a standard PA task, it is difficult to differentiate between these two possibilities. To define the underlying behavioral mechanism in our animals, we modified the PA task so that during the acquisition trial all animals were required to attain a specific level of performance (i.e., required to remain on the platform for 2 min after initial entry into the dark chamber). In this way, we could monitor failure of response inhibition (i.e., prolonged total learning time) as well as equalize "learning" across groups. Any group showing significantly increased total learning time could thus be said to be demonstrating a failure of response inhibition, and any performance deficits shown by this group on the retention trial could be attributed to failure of response inhibition. On the other hand, if initial learning time were similar among all groups, then retention deficits would be attributed to a failure in either consolidation or retrieval of memory, since initial acquisition of the task was similar.

Acquisition learning as assessed by total time to initial criterion was similar among all experimental groups, suggesting that neither disruption of septohippocampal cholinergic system nor the presence of HSI disrupts short-term information processing. This finding is not particularly surprising since other investigators have demonstrated that, when intratrial intervals are short, animals with damage to the hippocampus (including man) are quite capable of performing memory tasks (Milner et al., 1968). This suggests that normal hippocampal circuitry is not essential for this type of behavior.

During the initial acquisition trial, the MS/HSI group made more partial reentries than the other groups, which may be a reflection of their generalized hyperactivity. This effect was not found in the MS/Gx group, suggesting that the changes induced by HSI in the hippocampal circuitry were responsible for this finding.

Retention of the initial association was clearly impaired in the MS/HSI group when compared to the other three experimental groups. Since the MS/HSI animals were initially able to learn the task and thus withhold their responses as well as the other groups, we feel that this impairment was most likely secondary to a failure in the consolidation and/or retrieval of information rather than failure of response inhibition. A similar failure in working memory has been observed utilizing a radial eight-arm maze (RAM) paradigm (Harrell et al., 1983). The "normalization" of behavior in the MS/Gx animals is further evidence that, at least in male rats, HSI adversely effects PA behavior.

Forebrain NE has been suggested to play an integral role in reactivity to novel objects (i.e., neophobia) and novel tastes (i.e., gustatory neophobia). Previous studies (Mason et al., 1978; Tombaugh et al., 1983) have found an inverse relationship between NE levels and gustatory neophobia, wherein animals depleted of forebrain NE demonstrated enhanced gustatory neophobia. In our study, the two groups demonstrating deficits in gustatory neophobia were those with the highest concentrations of dorsal hippocampal NE (i.e., MS/HSI and Gx). This suggests that hippocampal NE is strongly linked to gustatory neophobia.

Lesions of the MS region have been reported to produce both an increase and a decrease (Donovick and Wakeman, 1969) in open field activity, as well as to produce no effects in this behavior (Kohler and Srebro, 1980). Our results suggest that hippocampal NE concentration may influence this behavior since 
animals with $\mathrm{HSI}$ were significantly more active than MS/Gx animals. Previous studies have demonstrated increased exploratory behavior, rearings, and changes in patterns of ambulation following infusion of $\mathrm{NE}$ into the hippocampus (Flicker and Geyer, 1982). In light of this study and our present findings, it is likcly that hippocampal NE may be an important factor in determining the activity levels of animals after MS/HSI lesions and may account for the discrepancies among previous studies.

The results of the present experiments, as well as of our previous studies, suggest that HSI can influence a broad range of behaviors. Traditionally, theories of learning and memory have emphasized the roles of both the hippocampus and cholinergic systems in the creation of memory traces (Deutsch, 1971; Leslie et al., 1985; Zornetzer, 1987). Recent data, however, suggest that the interaction of cholinergic and noradrenergic systems may be important for normal learning/memory processes (Leslie et al., 1985; Collier et al., 1988). Thus, Decker and Gallagher (1987) have found that in NE-depleted rats the administration of scopolamine, a muscarinic antagonist, facilitates the disruption of maze performance, while Sara et al. (1987) have demonstrated that clonidine, which decreases the release of $\mathrm{NE}$, improves mcmory processes in rats with cholinergic denervation of the hippocampus. Several studies (e.g., Vizi, 1980; Moroni et al., 1983) have demonstrated that NE inhibits the release of ACh. Furthermore, $\alpha_{2}$-receptors have also been found to be associated with GABAergic neurons and to increase the release of GABA (Pittaluga and Raiteri, 1987). One could therefore hypothesize that, in the setting of hippocampal cholinergic denervation, where there is a relative increase of NE (through either sympathetic ingrowth or spared central NE fibers), this "increased" level of NE either directly or indirectly through GABAergic interneurons inhibits the release of ACh from cholinergic intraneurons and/or spared cholinergic terminals. This decrease in ACh may in turn lead to poorer performance.

Hence, the balance between NE and ACh may be extremely important for learning/memory. This contention is further supported by work from our laboratory, demonstrating that phentolamine (an $\alpha$-adrenergic antagonist) treatment normalizes maze behavior in animals with HSI (Harrell et al., 1990b), and by recent work from other laboratories (Sara, 1989; Spangler et al., 1990). The mechanism by which this reversal occurs is unknown.

In summary, HSI is a neuronal reorganization that induces functional alterations within the CNS. Since it can be manipulated, controlled for, and reliably replicated, HSI serves as a valuable model system for studying the mechanisms by which neuronal reorganizations affect CNS function. We have shown that HSI results in a change in the relative content of $\mathrm{ACh}$ and $\mathrm{NE}$ in the hippocampus and, probably as a result of this, brings about changes in learned and spontaneous behaviors by mechanism(s) as yet unknown. The understanding of these mechanisms may lead to a better appreciation of the functional changes that occur as a consequence of neuronal reorganizations in neurodegenerative diseases such as Alzheimer's disease (Geddes et al., 1985; Booze et al., 1987; Hyman et al., 1987; Cotman and Anderson, 1988) or CNS injury (Cotman, 1985).

\section{References}

Barker DJ, Howard AJ, Gage FH (1984) Functional significance of sympathohippocampal sprouting: changes in single cell spontaneous activity. Brain Res 291:357-363.

Bignami G (1987) Avoidance methods in neurobehavioral toxicity assessments. Zentralbl Bakteriol [B] 185:36-47.
Black AH, Nadel L, O'Keefe J (1977) Hippocampal function in avoidance learning and punishment. Psychol Bull 84:1107-1129.

Booze RM, Gutman GR, Davis JN (1987) Catecholamine axonal morphology in Alzheimer's disease: examination of the sympathetic ingrowth hypothesis. Soc Neurosci Abstr 13:437.

Collier TJ, Gash DM, Sladek JR (1988) Transplantation of norepinephrinc ncurons into aged rats improves performance of a learned task. Brain Res 448:77-87.

Cotman CW, ed (1985) Synaptic plasticity. New York: Guilford.

Cotman CW, Anderson KJ (1988) Synaptic plasticity and functional stabilization in the hippocampal formation: possible role in Alzheimer's disease. In: Advances in neurology, Vol 47, Functional recovery in neurological disease (Waxman SG, ed), pp 313-335. New York: Raven.

Crutcher K, Kesner R, Novak JM (1983) Medial septal lesions, radial arm maze performance and sympathetic sprouting: a study of recovery of function. Brain Res 262:91-98.

Crutcher KA (1987) Sympathetic sprouting in the central nervous system: a model for studies of axonal growth in the mature mammalian brain. Brain Res Rev 12:203-233.

Curzon G, Hutson PII, Kennett GA, Marcou M, Gower A, Tricklebank MD (1986) Characteristics of analgesias induced by brief or prolonged stress. Ann NY Acad Sci 467:93-103.

Decker MW, Gallagher M (1987) Scopolamine-disruption of radial arm maze performance: modification by noradrenergic depletion. Brain Res 417:59-69.

De la Torre JC, Surgeon JW (1976) Histochemical fluorescence of tissue and brain monoamines: results in 18 minutes using the sucrosephosphate-glyoxylic acid (SPG) method. Neuroscience 1:451-454.

Deutsch JA (1971) The cholinergic synapse and the site of memory. Science 174:788-794.

Donovick PJ, Wakeman KA (1969) Open-field luminance and septal hyper-emotionality. Anim Behav 17:186-190.

Douglas RJ (1967) Hippocampus and behavior. Psychol Bull 67:416422.

Dunn LT, Everitt BJ (1987) The effects of lesions to noradrenergic projections from the locus coeruleus and lateral tegmental cell groups on conditioned taste aversion in the rat. Behav Neurosci 101:409422.

Flicker C, Geyer MA (1982) Behavior during hippocampal microinfusions. I. Norepinephrine and diversive exploration. Brain Res Rev 4:79-103.

Fonnum F (1969) Radiochemical microassay for the determination of choline acetyltransferase and acetylcholinesterase activities. Biochem J 115:465-472.

Galey D, Durkin T, Sifakis G, Kempf E, Jaffard R (1985) Facilitation of spontaneous and learned spatial behaviors following 6-hydroxydopamine lesions of the lateral septum: a cholinergic hypothesis. Brain Res 340:171-174.

Gallagher M, Burwell RD (1989) Relationship of age-related decline across several behavioral domains. Neurobiol Aging 10:691-708.

Geddes JW, Monaghan DT, Cotman CW, Lott IT, Kim RC, Chui HC (1985) Plasticity of hippocampal circuitry in Alzheimer's disease. Science 230:1179-1181.

Harrell LE, Barlow TS (1986) The superior cervical ganglia and learning of a spatial memory task. Physiol Behav 37:419-421.

Harrell LE, Parsons DS (1988) The role of gender in the behavior effects of peripheral sympathetic ingrowth. Exp Neurol 99:315-325.

Harrell LE, Barlow TS, Davis JN (1983) Sympathetic sprouting and recovery of a spatial behavior. Exp Neurol 82:379-390.

Harrell LE, Haring JH, Davis JN (1986) Peripheral sympathetic ingrowth can alter metabolic activity within the hippocampal formation. Exp Neurol 91:622-627.

Harrell LE, Goyal M, Parsons DS, Peagler A (1990a) The effect of gonadal steroids on the behavioral and biochemical effects of hippocampal sympathetic ingrowth. Physiol Behav 48:507-513.

Harrell LE, Peagler AD, Parsons DS (1990b) Adrenoreceptor antagonist treatment influences recovery of learning following medial septal lesions and hippocampal sympathetic ingrowth. Pharmacol Biochem Behav 35:21-28.

Heybach JP, Coover GD, Lints CE (1978) Behavioral effects of neurotoxic lesions of the ascending monoamine pathways in the rat brain. J Comp Physiol Psychol 92:58-70.

Huston JP, Oitzl MS (1989) The relationship between reinforcement and memory: parallels in the rewarding and mnemonic effects of the neuropeptide substance P. Neurosci Biobehav Rev 13:171-180. 
Hyman BT, Kromer BA, Van Hoesen GW (1987) Reinnervation of the hippocampal perforant pathway zone in Alzheimer's disease. Ann Neurol 21:259-267.

Kimble DP (1968) Hippocampus and internal inhibition. Psychol Bull 70:285-295.

Kimble DP, Anderson S, Bremiller R, Danna E (1979) Hippocampal lesions, superior ccrvical ganglia removal and behavior in rats. Physiol Behav 22:461-466.

Kohler C, Srebro B (1980) Effects of lateral and medial septal lesions on exploratory behavior in the albino rat. Brain Res 182:423-440.

Lamour Y, Bassant MH, Robert A, Joly Y (1989) Septo-hippocampal neurons in the aged rat: relation between their electrophysiological and pharmacological properties and behavioral performances. Neurobiol Aging 10:181-186.

Leslie FM, Loughin SE, Sternberg DB, McGaugh JL, Young LE, Zornetzer SF (1985) Noradrenergic changes and memory loss in aged mice. Brain Res 359:292-299.

Livesey PJ, Bell JA, Manyam V (1984) Disruption of passive avoidance learning in the rat by electrical stimulation of the hippocampus. Behav Neurosci 98:567-583.

Lowry OII, Rosebrough NJ, Farr AC, Randall PJ (1951) Protcin measurement with the Folin phenol reagent. J Biol Chem 193:265-275.

Madison R, Davis JN (1983) Regulation of hippocampal sympathetic ingrowth: role of afferent input. Brain Res 270:1-9.

Mason ST, Roberts DCS, Fibiger HC (1978) Noradrenaline and neophobia. Physiol Behav 21:353-361.

Milner B, Corkin S, Teuber HL (1968) Further analysis of hippocampal amnesic syndrome: 14 year follow-up study of H.M. Neuropsychologia 6:215-234.

Moroni F, Tanganelli S, Antonelli T, Carla V, Bianchi C, Beani L (1983) Modulation of cortical acetylcholine and $\gamma$-aminobutyric acid release in freely moving guinea pigs: effects of clonidine and other adrenergic drugs. J Pharmacol Exp Ther 227:435-440.

Naik NT (1963) Technical variations in Koelle's histochemical method for demonstrating cholinesterase activity. Q J Microsc Sci 104: 89-100.
Pepeu G, Casamenti F, Pedata F, Cosi C, Pepeu IM (1986) Are the neurochemical and behavioral changes induced by lesions of the nucleus basalis in the rat a model of Alzheimer's disease? Prog Neuropsychopharmacol Biol Psychiatry 10:541-551.

Pittaluga A, Raiteri M (1987) GABAergic nerve terminals in rat hippocampus possess $\alpha$-adrenoreceptors regulating GABA release. Neurosci Lett 76:363-367.

Rigter H, van-Riezen H (1979) Pituitary hormones and amnesia. Curr Dev Psychopharmacol 5:67-124.

Sanger DJ, Joly D (1990) Psychopharmacological strategies in the search for cognition enhancers. Pharmacopsychiatry [Suppl 2] 23:7074.

Sara SJ (1989) Noradrenergic-cholinergic interaction: its possible role in memory dysfunction associated with senile dementia. Arch Gerontol Geriatr [Suppl] 1:99-108.

Sara SJ, Maho C, Ammassari M (1987) Clonidine reverses learning deficits after partial fornix section. Soc Neurosci Abstr 13:184.7.

Slangen JL, Earley B, Jaffard R, Richelle M, Olton DS (1990) Behavioral models of memory and amnesia. Pharmacopsychiatry [Suppl 2] 23:81-83.

Spangler EL, Wenk GL, Chachich ME, Smith K, Ingram DK (1990) Complex maze performance in rats: effects of noradrenergic depletion and cholinergic blockade. Behav Neurosci 104:410-417.

Tombaugh TN, Pappas BA, Roberts DCS, Vickers GJ, Szostak C (1983) Failure to replicate the dorsal bundle extinction effect: telencephalic norepinephrine depletion does not reliably increase resistance to extinction but does augment gustatory neophobia. Brain Res 261:231-242.

Vizi ED (1980) Modulation of cortical release of acetylcholine by noradrenaline released from nerves arising from the rat locus coeruleus. Neuroscience 5:2139-2144.

Winer BJ (1962) Statistical principles in experimental design. New York: McGraw-Hill.

Zornetzer S (1987) Catecholamine system involvement in age-related memory dysfunction. Ann NY Acad Sci 444:242-254. 